\title{
Cerebello-Cerebral Connectivity Deficits in Friedreich Ataxia
}

\author{
Andrew Zalesky ${ }^{1}$, Hamed Akhlaghi ${ }^{2}$, Louise A. Corben ${ }^{3}$, John L. Bradshaw ${ }^{4}$, \\ Martin B. Delatycki ${ }^{3,5}$ Elsdon Storey ${ }^{6}$, Nellie Georgiou-Karistianis ${ }^{4}$, and Gary F. Egan ${ }^{7}$ \\ ${ }^{1}$ Melbourne Neuropsychiatry Centre, The University of Melbourne, Victoria 3010, \\ Australia. \\ ${ }^{2}$ Howard Florey Institute, Florey Neuroscience Institutes, Victoria 3010, Australia. \\ ${ }^{3}$ Bruce Lefroy Centre, Murdoch Childrens Research Institute, Victoria 3052, Australia. \\ ${ }^{4}$ School of Psychology and Psychiatry, Monash University, Victoria 3800, Australia. \\ ${ }^{5}$ Department of Clinical Genetics, Austin Health, Heidelberg, Victoria 3084, Australia. \\ ${ }^{6}$ Department of Medicine (Neuroscience), Monash University (Alfred Hospital Campus), \\ Victoria, Australia. \\ ${ }^{7}$ Monash Biomedical Imaging, Monash University, Victoria 3800, Australia.
}

Running Title: Connectivity Deficits in Friedreich Ataxia

Corresponding Author: Melbourne Neuropsychiatry Centre

Level 3, Alan Gilbert Building

The University of Melbourne

Victoria 3010, Australia

Email: azalesky@unimelb.edu.au

Phone: (+613) 90357747 


\section{Abstract}

Brain pathology in Friedreich ataxia is characterized by progressive degeneration of nervous tissue in the brainstem, cerebellum and cerebellar peduncles. Evidence of cerebral involvement is however equivocal. This brain imaging study investigates cerebello-cerebral white matter connectivty in Friedreich ataxia with diffusion MRI and tractography performed in 13 individuals homozygous for a GAA expansion in intron 1 of the frataxin gene and 14 ageand gender-matched control participants. New evidence is presented for disrupted cerebello-cerebral connectivity in the disease, leading to secondary effects in distant cortical and subcortical regions. Remote regions affected by primary cerebellar and brainstem pathology include the supplementary motor area, cingulate cortex, frontal cortices, putamen and other subcortical nuclei. The connectivity disruptions identified provide an explanation for some of the non-ataxic symptoms observed in the disease and support the notion of reverse cerebellar diaschisis. This is the first study to comprehensively map white matter connectivity disruptions in Friedreich ataxia using tractography, connectomic techniques and super-resolution track density imaging.

\section{Keywords}

Friedreich ataxia, diffusion-weighted imaging, white matter, connectome, connectivity. 


\section{Introduction}

Friedreich ataxia (FRDA) is an autosomal recessive disease with a prevalence of about 1 in 30,000 (Schulz et al, 2009). In about $98 \%$ of cases, FRDA is caused by homozygosity for an intronic GAA repeat expansion in the frataxin (FXN) gene on chromosome 9q13 (Klockgether, 2000).

The most prominent pathology associated with FRDA is degeneration of the dorsal root ganglia, peripheral nerves, spinal cord, spinocerebellar and corticospinal tracts, and cerebellum (Dürr et al, 1996; França et al, 2009). Magnetic resonance imaging (MRI) and computed tomography (CT) of the central nervous system has confirmed atrophy of these structures (Mascalchi et al, 1994; Mascalchi \& Vella, 2012; Omerod et al, 1994; Junck et al, 1994; Wüllner et al, 1993), with one study specifically reporting atrophy of the superior cerebellar peduncles (Akhlaghi et al, 2011).

Evidence of cerebral pathology in FRDA is scant (França et al, 2009), despite the presence of symptoms that cannot be directly attributed to the ataxia per se, such as cognitive dysfunction (Corben et al, 2006; Wollmann et al, 2004), information processing speed deficits (Hart et al, 1985) and psychomotor slowing (White et al, 2000). The cognitive symptoms in FRDA could be consequences of axonal loss within white matter connections supporting cerebro-cerebellar loops (Klopper et al, 2011; Mantovan et al, 2006; Wollmann et al, 2004). In particular, interruption to the afferent and efferent connections linking the cerebellum with prefrontal, posterior parietal, superior temporal and limbic cortices may result in cerebellar diaschisis. The reverse cerebellar diaschisis hypothesis posits that functional changes in the cortex are secondary 
to a loss of anatomical connectivity with the cerebellum, resulting in the cerebellar cognitive affective syndrome (Schmahmann \& Sherman, 1998).

Evidence for cerebellar diaschisis in FRDA is equivocal. Functional MRI and PET imaging studies have revealed abnormal changes in cortical activation (Georgiou-Karistianis et al, 2012; Ginestroni et al, 2012; Mantovan et al, 2006) and cortical glucose metabolism (Gilman et al, 1990). While these functional findings are consistent with disrupted cerebello-cerebral connectivity, they do not exclude the alternative hypothesis of primary dysfunction in cortical activation.

Diffusion MRI and white matter tractography (Catani et al, 2002; Conturo et al, 1999; Gong et al, 2009a) are ideally suited to investigate the integrity of major cerebello-cerebral pathways. Despite this, diffusion MRI studies in FRDA have focussed on voxel-wise comparison of various diffusion measures (see Table 1). No previous study has performed tractography to investigate brain connectivity in the disease. Tractography coupled with network modeling techniques have provided novel insight in several other brain disorders (Bassett \& Bullmore, 2009).

The aim of the present study was to use novel tractography techniques to comprehensively map cerebello-cerebral connectivity in FRDA. It was hypothesized that white matter pathways, linking the cerebellum with the cortex, would show evidence of disruption in FRDA compared to controls.

To test this hypothesis, tractography was performed in 13 individuals homozygous for a GAA expansion in intron 1 of the FXN gene and 14 control participants matched for age and gender. The tractography results were analyzed using novel connectivity methods, which yielded the first evidence of disrupted cerebello-cerebral connectivity in FRDA as well as high-resolution images of the associated white matter degeneration. 


\section{Materials and Methods}

\subsection{Participants}

Thirteen adults homozygous for a GAA repeat in intron 1 of the $F X N$ gene participated in this study (6 males, mean age: $35.5 \pm 9.4$ years). Fourteen control participants matched for age and gender also participated (9 males, mean age: 33.7 \pm 7.9 years). Age an gender matching is crucial (Gong et al, 2009b; Gong et al, 2011). Exclusion criteria for participants included a current or lifetime history of diagnosable medical, major psychiatric or neurologic conditions (other than FRDA). All participants completed the Beck Depression Inventory (BDI) (Beck et al, 1961) to assess for the presence of symptoms related to depression. Participants also completed the National Adult Reading Test (NART), which provides an estimate of pre-morbid intelligence (Nelson et al, 1992). There was no significant difference in BDI scores between the groups, however a one-way ANOVA demonstrated a significant $\left(F_{(1,25)}=14.51, p<0.001\right)$ difference between groups on the NART (see Table 2). Clinical severity in individuals with FRDA was evaluated by the Friedreich Ataxia Rating Scale (FARS) (Lynch et al, 2006; Subramony et al, 2005).

Approval for this study was obtained from the Royal Children's Hospital (HREC 26147 C) and Melbourne Health (National Neuroscience Foundation, HREC 2007.027) Human Research Ethics Committees. All participants gave informed, written consent in accordance with the Declaration of Helsinki.

\subsection{Diffusion Magnetic Resonance Imaging}

A series of diffusion-weighted magnetic resonance images of brain anatomy were acquired in each participant using a Siemens Trio Magnetom 3 Tesla system (Siemens, Erlangen) located at the Murdoch Childrens Research 
Institute, Melbourne, Australia. Sixty diffusion-weighted volumes were acquired using a spin-echo EPI sequence with the following parameters: b-value, 2000 $\mathrm{s} / \mathrm{mm}^{2} ; 64$ consecutive axial slices of thickness $2 \mathrm{~mm}$; $128 \times 128$ image matrix with an in-plane voxel resolution of $2 \times 2 \mathrm{~mm}$; field of view, $25.6 \times 25.6 \mathrm{~cm}$; repetition time, 7720 ms; echo time, 88 ms; flip angle, 90 degrees. Ten nondiffusion-weighted volumes were also acquired. Participants were not scanned in any particular order.

The signal-to-noise ratio (SNR) was estimated for each individual using the "difference method" (Dietrich et al, 2007). This involved voxel-by-voxel summation of the signal between the last two non-diffusion-weighted volumes and averaging the resulting sum over a small region-of-interest (ROI) positioned in deep cerebral white matter, yielding the quantity $m_{\text {sum }}$. Similarly, the difference between the last two non-diffusion-weighted volumes was determined and the standard deviation computed for the resulting difference over voxels comprising the same white matter ROI, yielding the quantity $s_{\text {diff }}$ The SNR was defined as the quotient of these two quantities, $m_{\text {sum }} / s_{\text {diff }}$, multiplied by a factor of $1 / \sqrt{2}$ (Dietrich et al, 2007). The SNR did not significantly differ between groups (FRDA: $21.6 \pm 2.0$, Controls: $22.9 \pm 1.4 ; t_{(25)}=1.9, p=0.07$ ).

The extent of intra-acquisition head movement was estimated using the affine transformation matrices resulting from eddy current correction (see Data Preprocessing and Image Registration). Head movement did not significantly differ between groups for any of the three displacement parameters or any of the three rotation parameters. The displacements in all of the three directions never exceeded $1.0 \mathrm{~mm}$ for any volume of any participant. The rotations about all of the three axes never exceed $3.5^{\circ}$ for any volume of any participant, except for one individual with FRDA, who showed no more than $4.6^{\circ}$ of rotation. 


\subsection{Data Preprocessing and Image Registration}

Eddy current correction followed by estimation of a fractional anisotropy (FA) image for each participant was achieved using FDT, v2.0, the diffusion toolkit within FSL (FMRIB's Software Library; www.fmrib.ox.ac.uk/fsl). The eddy current correction step minimized distortions induced by eddy currents and also aligned each diffusion-weighted volume to the first non-diffusion weighted volume to correct for simple intra-acquisition head movement. Rotations applied to the diffusion-weighted volumes were also applied to the corresponding gradient directions (Leeman \& Jones, 2009). FA was estimated using weighted linear least squares fitted to the log-transformed data.

A custom FA template was constructed in MNI152 space using an iterative registration scheme (Tyszka et al., 2011). For the initial iteration, the skullstripped FA images were registered using FLIRT, v5.5, 12 degrees of freedom, (Jenkinson \& Smith, 2001) to the standard FA brain provided by FMRIB (1 mm isotropic). The average of all registered images became the template for the second iteration. This sequence was repeated for a total of three iterations to yield a final set of registered FA images and a final custom FA template. Figure 1 shows the average of the registered FA images in the FRDA and control groups. The registered FA images were carefully inspected for any irregularities. Consideration was given to applying a subsequent nonlinear registration step to further improve alignment. However, excessive warping became a concern after nonlinear registration. To be conservative, warping was therefore not applied, albeit at the expense of potentially risking a loss in sensitivity due to residual misalignment effects. 


\subsection{Fractional Anisotropy Between-Group Comparison}

To corroborate the anatomical location of previously reported FA decreases in individuals with FRDA (see Table 1), a voxel-wise between-group comparison of FA was first performed. The $t$-statistic was used to test the null hypothesis of equality in the mean value of FA between groups at every voxel comprising a liberal brain mask. The mask included the cerebellum, brainstem and cerebral gray and white matter. Permutation testing was then used to ascribe a corrected $p$-value to voxel clusters based on their extent (Nichols \& Holmes, 2001). This step was performed using Randomise, v2.9, with a primary $t$-statistic threshold of 3 and a total of 10,000 permutations.

Consideration was given to using tract-based spatial statistics (TBSS) (Smith et al, 2006). However, this approach standardly employs nonlinear registration, which we were cautious of applying due to the above concerns about excessive/erroneous warping in our FA volumes. Furthermore, recent evidence suggests skeleton-based methods are rotationally variant, leading to considerable orientation bias (Edden \& Jones, 2011), and the 'align-with-maxFA' strategy inherent to TBSS may improve concordance in FA values but does not necessarily improve anatomical concordance (Zalesky, 2011a).

\subsection{Tractography}

Reduced FA in the FRDA group was isolated to a bilateral cluster spanning parts of the brainstem, dentate nucleus and cerebellar peduncles (see Results and Figure 1). Probabilistic white matter tractography (Behrens et al, 2007) was used in an exploratory manner to identify the distant brain regions and upstream fiber bundles affected by this primary white matter degeneration. Tractography was performed in native space. To this end, the cluster corresponding to the between-group difference in FA was first registered to each participant's native 
space using the inverse of the affine transform resulting from FA registration. A total of 5000 streamlines were then initiated from each voxel ( $2 \mathrm{~mm}$ isotropic) comprising the cluster. This was increased to 32,000 streamlines per voxel for track density imaging to enable reliable super-resolution sampling of streamline counts (Calamante et al, 2010). Tractography was performed using ProbtrackX, build 419 , with a step length of $200 \mu \mathrm{m}$, the default curvature threshold of 0.2 (equivalent to \pm 80 degrees). Streamlines were initiated from the centre of each voxel.

When mapped to participants' native space, the volume of the FA cluster did not significantly differ between groups (FRDA: $22.1 \pm 1.7 \mathrm{cc}$, Controls: $25.9 \pm 13.9$ $\left.c c ; t_{(25)}=1.0, p=0.3\right)$. This ensured that the total number of streamlines generated per participant also did not differ between groups.

\subsection{Connectomic Mapping}

While evidence for white matter degeneration was confined to the brainstem, dentate nucleus and cerebellar peduncles, these primary sites of pathology may disrupt cerebello-cerebral connectivity and thereby impede cerebellar communication with remote cortical structures. The purpose of connectomic mapping was to identify the distant brain regions (if any) that were affected by the primary white matter pathology. To identify these remote structures, the 90 cortical and subcortical nodes comprising the Automated Anatomical Labeling (AAL) template (Tzourio-Mazoyer et al, 2002) were used as candidate structures. The 90 AAL nodes were registered to each participant's native space using the inverse of the affine transform resulting from FA registration. The total number of streamlines reaching each node was then enumerated, which reflected the extent of putative connectivity between the primary cerebellar pathology and each distant cortical and subcortical node. The $t$-statistic was used to separately test the null hypothesis of equality in the mean streamline 
count between groups for each node. The false discovery rate was used to correct for the 90 multiple comparisons (Genovese et al, 2002).

\subsection{Track Density Imaging}

Track density imaging (TDI) is a relatively common approach to visualize and perform voxel-wise comparisons of tractography results (e.g. Barrick et al, 2005; Bozzali et al, 2011). The intensity of a voxel in a track density image is modulated by the number of streamlines passing through it, reflecting the number of streamlines per unit volume. Recently, Calamante and colleagues (Calamante et al, 2010) demonstrated the super-resolution property of TDI and validated this property with mouse brain histology (Calamante et al, 2012). The super-resolution property refers to binning the streamline counts at a higher resolution than the acquired imaging voxels. Super-resolution TDI has been shown to yield white matter images with excellent anatomical contrast (Calamante et al, 2011).

In this study, TDI was performed at a resolution of $500 \mu \mathrm{m}$, four times finer than the native imaging resolution. The streamline step length was set to $200 \mu \mathrm{m}$ to ensure a streamline that is pointing perpendicular to the face of a superresolution voxel will necessarily have two successive points along its trajectory that will land in that voxel. Length scaling (Calamante et al, 2011) was not performed because all streamlines were initiated from a local cluster, and thus the disproportionately high sampling of long fiber bundles seen with whole-brain tractography was not a concern (Zalesky et al, 2009). Super-resolution TDI was performed using ProbtrackX by specifying the seed mask (between-group difference in FA) in the desired super resolution. Five hundred streamlines were initiated from each $500 \mu \mathrm{m}$ isotropic voxel comprising the seed mask, which is equivalent to 32,000 streamlines per $2 \mathrm{~mm}$ isotropic. This number was found to be more than adequate to yield a sufficiently dynamic range of track density counts. 
The track density image for each participant was registered to superresolution $\mathrm{MNI}$ space using the affine matrices resulting from FA registration. The average of the registered track density images was then used as a custom TDI template. Using FLIRT, v5.5, 12 degrees of freedom, (Jenkinson \& Smith, 2001) the super-resolution track density images were registered to this custom template. Only one pass of this iterative registration process was performed due to the increased computational burden associated with super-resolution registration.

Finally, the super-resolution track density images were binarized using a range of thresholds to yield estimates for the total volume of the cerebellocerebral pathways in each participant. A super-resolution voxel was set to unity if its track density exceeded the predefined threshold, otherwise it was set to zero. Volume was then estimated by summing the volumes encapsulated by each super-resolution voxel set to unity. A range of thresholds were investigated. For each threshold, the $t$-statistic was used to test the null hypothesis of equality in the mean volume between groups. This bears resemblance to the approach proposed by Barrick and colleagues (Barrick et al, 2005).

\subsection{Clinical Correlations}

Pearson's product-moment correlation was used to assess the significance of any associations between clinical variables and neuroimaging measures in the individuals with FRDA. The clinical variables considered were the FARS score, age of disease onset, duration of disease and GAA repeat length (smaller allele), which is known to correlate with age of onset and severity. These clinical variables were each separately correlated with two neuroimaging measures: i) the volume of the cerebello-cerebral pathways, as estimated with TDI; and, ii) the number of streamlines reaching the cortical and subcortical regions found to show a significant between-group difference. 


\section{Results}

Figure 1 shows the average of the registered FA images in the FRDA and control groups. All images are shown in radiological convention (left on right). No qualitative between-group differences in mean FA were evident. Statistical testing revealed a single cluster spanning portions of the brain stem, dentate nucleus and cerebellar peduncles with significantly reduced FA in the FRDA group compared to controls ( $p<0.001$, corrected; see Figure 1 ). This is consistent with the location of white matter degeneration reported in previous diffusion MRI studies in the disease (see Table 1).

Connectomic mapping was performed using white matter tractography to identify the distant brain regions between which cerebello-cerebral connectivity was impeded as a consequence of the primary cerebellar and brainstem pathology. Fourteen of the 90 cortical and subcortical regions considered $(\approx 16 \%)$ were connected with significantly fewer streamlines in the individuals with FRDA ( $p<0.05$, false discovery rate corrected for 90 independent tests). These aberrantly connected regions as expected included the supplementary motor area, but also the cingulate cortex, frontal cortices and numerous subcortical nuclei. (See Figure 2 for a schematic representation and Table 3 for group-averaged streamline counts and corresponding standard errors and $p$ values.) This result points to the importance of conceptualizing FRDA pathology not only in terms of the well-known primary sites of white matter degeneration, but also in terms of the spatially distributed network of cortical and subcortical regions that are likely to be secondarily affected through connectivity deficits. While between-group tractography differences are generally difficult to ascribe to a pathological mechanism (Jbabdi \& Johansen-Berg, 2012), the present finding is robust, with large effect sizes ranging between 4.0 and 7.3 (Cohen's d), and thus is likely to be reproducible. 
Super-resolution TDI was performed to visualize the structure of major cerebello-cerebral pathways. Figure 3 shows representative slices from the group-averaged track density images (500 $\mu \mathrm{m}$ isotropic). The particular slices shown have been chosen to represent four white matter structures: anterior thalamic radiation (column $A$ ), brainstem and dentate nucleus $(B)$, middle cerebellar peduncle (C) and corticospinal tract (D). In Figure 3, the green box superimposed on the whole-brain structural image serves to indicate the approximate anatomical region captured by each representative slice. Appreciable between-group differences are visually evident when comparing corresponding slices from the group-averaged track density images.

Specifically, images are markedly fainter in the FRDA group, particulary along the periphery of the white matter structures represented. The images are slightly left-right asymmetric, most likely because the cluster from which the streamlines were initiated was also asymmetric, although the presence of natural left-right morphological asymmetries (Galaburda et al, 1978; Yakovelv \& Rakic, 1996) cannot be discounted.

The total volume of the cerebello-cerebral pathways was estimated in each participant by applying a range of binarizing thresholds to the super-resolution track density images. Thresholds ranged between 1000 and 12,000 streamlines, evaluated in increments of 1000. Figure 4 (panel A) plots the group-averaged volume estimates as a function of the binarizing threshold. Volume in the individuals with FRDA (red plot) was significantly lower than in the controls (blue plot) for all the thresholds considered $(p<0.001)$. Additionally, Figure 4 shows the group-averaged anatomical location of the binary clusters from which volume was estimated for two representative thresholds: 4000 (panel B) and 6000 (panel C). The FRDA cluster (red) is clearly encapsulated by the wider margins of the cluster corresponding to the control group (blue).

A negative correlation was found in the individuals with FRDA between the age of disease onset and volume (Threshold of 8000: $r=-0.6, p=0.02 ; 10,000$ : 
$r=-0.6, p=0.01 ; 12,000: r=-0.7, p=0.01)$. Scatter plots representing this association are shown in Figure 5. However, the association did not reach significance when actual age was included as a nuisance covariate, or when correction was performed for the multiple independent correlations evaluated. No other significant correlations were found.

\section{Discussion}

This is the first study to map white matter connectivity disruptions in individuals with FRDA. Neuroimaging techniques provided evidence for brain pathology extending beyond the well-known cerebellar and brainstem degeneration. In particular, several distant cortical and subcortical regions were found to be aberrantly connected with the cerebellum in FRDA. As expected, these included regions associated with motor movement, inhibition and planning: namely, the supplementary motor area, putamen and pallidum. However, non-motor regions, not typically associated with pathology in FRDA, were also implicated, including the cingulate cortex, hippocampus and frontal cortices, providing evidence of disruption to afferent and efferent white matter pathways linking them to the cerebellum and brainstem.

Deficits in cerebellar connectivity to regions associated with executive function and cognition provide a putative biological basis for the non-motor symptoms sometimes seen in individuals with FRDA (Corben et al, 2010, 2011a, 2011b, 2011c; Klopper et al, 2011; Wollmann et al, 2004) and increased response times (Hart et al, 1985; White et al, 2000). The present findings suggest that these symptoms may be directly consequent to a progressive degradation of cerebellar connectivity with the cingulate and frontal cortices. This provides support for the notion of reverse cerebellar diaschisis in the disease (Schmahmann \& Sherman, 1998).

Significant correspondence can be observed between the regions implicated in this study and a recent functional MRI study undertaken in the same cohort 
(Akhlaghi et al, 2012). Functional MRI images were acquired during finger tapping tasks and significant between group differences in cortical activation were localized to the supplementary motor area (SMA), putamen and cerebellum as well as frontal and parietal regions. Evidence from two distinct neuroimaging modalities therefore points towards widespread cerebral involvement in FRDA pathology, both in terms of degenerating white matter pathways and aberrant cortical activation. Our diffusion MRI study supports the hypothesis that the aberrant activation patterns and decreased motor performance seen in the FRDA group during the finger tapping tasks were due to disrupted white matter pathways impeding visuomotor coordination between distant brain regions.

Tractography maps of cerebello-cerebral pathways were analyzed using two complementary techniques: tractography (Sporns et al, 2005) and superresolution TDI (Calamante et al, 2010). Tractography enabled precise localization of the distant cortical and subcortical regions affected by aberrant cerebello-cerebral connectivity. The structure of the corresponding pathways was then visualized with super-resolution TDI. In contrast, previous diffusionMRI studies in FRDA have focussed on characterizing white matter integrity using voxel-wise measures derived from the diffusion tensor, rather than mapping connectivity per se. Although the voxel-wise approach has been tremendously successful in identifying the primary sites of white matter degeneration in the disease, it has been unable to reveal any downstream cortical effects.

In this study, a significant reduction in the number of streamlines comprising cerebello-cerebral loops is presented as evidence for their disruption. The pathological mechanisms underlying this "disruption" are not well understood, but could potentially indicate demyelination or a reduction in the number, density and/or coherence of axonal projections (Zalesky et al, 2011). There is no evidence for demyelination in individuals with FRDA and immunocytochemistry 
suggests that axonal density (axons per unit area) is normal, at least in the dorsal root of the lumbar spinal cord, but myelin sheaths are thinner (Koeppen, 2011). The speed at which information can be transferred via cerebello-cerebral loops may therefore be diminished, since thinning of large myelinated fibers may result in a slowing of the conduction velocity of nerve pulses (Rushton, 1951), thereby delaying information transfer. This provides a potential explanation for the psychomotor slowing and processing speed deficits seen in FRDA (Corben et al, 2010, 2011a; Koeppen, 2011; White et al, 2000).

Investigations based on mouse models representative of FRDA (AIMahdawi et al, 2006; Puccio et al, 2001; Simon et al, 2004) have reported swelling and possible demyelination of large axons in the lumbar dorsal root ganglia, in addition to motor deficits and reductions in frataxin mRNA expression. However, detailed investigations of ultrastructural brain pathology in FRDA mouse models are scant, with most studies focusing on the hallmark cardiac and dorsal root ganglia degeneration. Others have even reported absence of any brain and spinal cord pathology (Al-Mahdawi et al, 2006). Utilizing mouse model studies to inform the pathological origins of the connectivity disruptions we have identified is therefore difficult. The degenerative processes observed in the dorsal root ganglia (i.e. thinning of myelin sheaths, swelling and the presence of vacuoles in neuronal cell bodies) may be ubiquitous and also underlie any degeneration in brain connectivity, however further neuroimaging studies are required to assess this hypothesis.

A significant reduction in the volume of the binarized track density images was found in individuals with FRDA, possibly indicating atrophy. This is in line with a recent study that derived atrophy estimates for specific white matter structures in individuals with FRDA and reported these estimates were correlated with clinical status (Pagani et al, 2010). The volume estimates derived in our study may vary appreciably compared to estimates yielded by manual tracing. Due to the distal fading associated with streamline tractography 
(Zalesky et al, 2009), areas more distant from the seed region are undersampled, since some streamlines inevitably terminate prematurely before reaching distant areas. As such, distant areas were the first to succumb to the binarizing threshold, whereas areas more proximal to the seed region survived. While the volume estimates reported in this study are suitable as a relative measure for between-group comparison, they should not be interpreted as an absolute indication of volume.

Several potential limitations should be noted. First, the sample size was relatively small. This was a limiting factor when investigating associations between clinical variables and neuroimaging measures in the FRDA group. While a negative correlation was found between age of disease onset and volume, this did not survive multiple comparisons correction or when actual age was included as a nuisance covariate. This negative correlation may indicate a compensatory effect in FRDA individuals with an earlier age of onset, possibly a consequence of ongoing brain maturation in adolescence. However, we are cautious about any interpretation due to the noted statistical limitations.

This study utilized state-of-the-art methods to investigate white matter connectivity, many of which require further validation. For example, while multiple fiber orientations were used to model complex fiber geometries, such as crossing fibers (Behrens et al, 2007), tractography is still an active area of research and current fiber tracking algorithms have numerous limitations (Jbabdi \& Johansen-Berg, 2012). Another methodological limitation is the arbitrary use of the AAL template to define cortical and subcortical regions-of-interest (i.e. nodes). Connectomic mapping can be influenced by the choice and number of nodes (Wig et al, 2011; Zalesky et al, 2010), and thus the present findings are contingent on the validity of the AAL template. Finally, the co-registration of super-resolution track density images remains an open issue.

In conclusion, this is the first study to investigate white matter connectivity in individuals with FRDA. The present findings suggest conceptualizing FRDA 
pathology not only in terms of well-known primary sites of white matter degeneration, but also in terms of a spatially distributed network of cortical and subcortical regions that are likely to be secondarily affected through connectivity deficits. The connectivity disruptions may provide an explanation for the nonmotor symptoms seen in the disease and support the notion of reverse cerebellar diaschisis. However, to establish this hypothesis, future work should assess whether connectivity deficits are correlated with the extent of non-motor symptoms in FRDA, such as depression (da Silva et al, 2012) and cognitive difficulties (Corben et al, 2011a; Georgiou-Karistianis et al 2012). Except for the Simon effect, which may be considered a non-motor sign, the cognitive functioning of our sample was not quantitatively characterized, thereby precluding this kind of correlation analysis.

Future work should also focus on investigating any correspondence with resting-state functional connectivity deficits (van den Heuvel et al, 2009; van den Heuvel \& Hulshoff Pol, 2010) and possible alterations in topological measures (Rubinov \& Sporns, 2010; He \& Evans, 2010; van den Heuvel et al, 2008).

\section{Funding}

This work was supported by funding from the Friedreich Ataxia Research Association (Australasia), Friedreich Ataxia Research Alliance (USA) and Murdoch Childrens Research Institute. Authors were supported by the Australian Research Council [DP0986320 to A.Z.]; the Melbourne Neuroscience Institute [Melbourne Neuroscience Institute Fellowship to A.Z.]; the University of Melbourne [Melbourne International Fee Remission Scholarship and Melbourne International Research Scholarship to H.A.], the National Health and Medical Research Council [Early Career Fellowship to L.A.C., Practitioner Fellowship to M.B.D., Research Fellowship to G.F.E]. 


\section{References}

Akhlaghi H, Corben L, Georgiou-Karistianis N, Bradshaw J, Storey E, Delatycki MB, Egan GF. 2011. Superior cerebellar peduncle atrophy in Friedreich's ataxia correlates with disease symptoms. Cerebellum. 10(1):81-87.

Akhlaghi H, Corben L, Georgiou-Karistianis N, Bradshaw J, Delatycki MB, Storey E, Egan GF. 2012. A functional MRI study of motor dysfunction in Friedreich's ataxia. Brain Res. 1471:138-154.

Al-Mahdawi S, Pinto RM, Varshney D, Lawrence L, Lowrie MB, Hughes S, Webster Z, Blake J, Cooper JM, King R, Pook MA. 2006 GAA repeat expansion mutation mouse models of Friedreich ataxia exhibit oxidative stress leading to progressive neuronal and cardiac pathology. Genomics. 88:580-590.

Barrick TR, Lawes IN, Hall MG, Clark CA. 2005. Quantification Of white matter pathway volume from diffusion tensor tractography. Proc Intl Soc Mag Reson Med. 13:1348.

Bassett DS, Bullmore ET. 2009. Human brain networks in health and disease. Current Opinion in Neurology. 22(4):340-347.

Beck AT, Ward CH, Mendelson M, Mock J, Erbaugh J. 1961. An inventory for measuring depression. Arch Gen Psychiatry. 4:561-571.

Behrens TE, Berg HJ, Jbabdi S, Rushworth MF, Woolrich MW. 2007. Probabilistic diffusion tractography with multiple fibre orientations: What can we gain? Neuroimage. 34(1):144-155.

Bozzali M, Parker GJ, Serra L, Embleton K, Gili T, Perri R, Caltagirone C, Cercignani M. 2011. Anatomical connectivity mapping: a new tool to assess brain disconnection in Alzheimer's disease. Neuroimage. 54(3):2045-2051. 
Calamante F, Tournier JD, Jackson GD, Connelly A. 2010. Track-density imaging (TDI): super-resolution white matter imaging using whole-brain track-density mapping. Neuroimage. 53(4):1233-1243.

Calamante F, Tournier JD, Heidemann RM, Anwander A, Jackson GD, Connelly A. 2011. Track density imaging (TDI): validation of super resolution property. Neuroimage. 56(3):1259-1266.

Calamante F, Tournier JD, Kurniawan ND, Yang Z, Gyengesi E, Galloway GJ, Reutens DC, Connelly A. 2012. Super-resolution track-density imaging studies of mouse brain: comparison to histology. Neuroimage. 59(1):286-296.

Catani M, Howard RJ, Pajevic S, Jones DK. 2002. Virtual in vivo interactive dissection of white matter fasciculi in the human brain. Neuroimage. 17:77-94.

Conturo TE, Lori NF, Cull TS, Akbudak E, Snyder AZ, Shimony JS, McKinstry RC, Burton H, Raichle ME. 1999. Tracking neuronal fiber pathways in the living human brain. Proc Natl Acad Sci USA. 96:10422-10427.

Corben LA, Akhlaghi H, Georgiou-Karistianis N, Bradshaw JL, Egan GF, Storey E, Churchyard AJ, Delatycki MB. 2011a. Impaired inhibition of prepotent motor tendencies in friedreich ataxia demonstrated by the simon interference task. Brain Cogn. 76(1):140-145.

Corben LA, Delatycki MB, Bradshaw JL, Churchyard AJ, Georgiou-Karistianis N. 2011b. Utilization of advance motor information is impaired in Friedreich ataxia. Cerebellum. 10(4):793-803.

Corben LA, Delatycki MB, Bradshaw JL, Horne MK, Fahey MC, Churchyard AC, Georgiou-Karistianis N. 2010. Impairment in motor reprogramming in Friedreich ataxia reflecting possible cerebellar dysfunction. J Neurol, 257(5):782-791.

Corben, LA, Georgiou-Karistianis N, Bradshaw JL, Hocking, DR, Churchyard AJ, Delatycki MB. 2011c. The Fitts task reveals impairments in planning and online 
control of movement in Friedreich ataxia: reduced cerebellar-cortico connectivity? Neuroscience. 192:382-390.

Corben LA, Georgiou-Karistianis N, Fahey MC, Storey E, Churchyard A, Horne M, Bradshaw JL, Delatycki MB. 2006. Towards an understanding of cognitive function in Friedreich ataxia. Brain Res Bull. 70(3):197-202.

da Silva CB, Yasuda CL, D'Abreu A, Cendes F, Lopes-Cendes I, França MC Jr. 2012. Neuroanatomical correlates of depression in Friedreich's ataxia: a voxelbased morphometry study. Cerebellum. In press.

Dietrich O, Raya JG, Reeder SB, Reiser MF, Schoenberg SO. 2007. Measurement of signal-to-noise ratios in MR images: influence of multichannel coils, parallel imaging, and reconstruction filters. J Magn Reson Imaging. 26(2):375-385.

Della Nave R, Ginestroni A, Tessa C, Salvatore E, Bartolomei I, Salvi F, Dotti MT, De Michele G, Piacentini S, Mascalchi M. 2008. Brain white matter tracts degeneration in Friedreich ataxia. An in vivo MRI study using tract-based spatial statistics and voxel-based morphometry. Neuroimage. 40(1):19-25.

Della Nave R, Ginestroni A, Diciotti S, Salvatore E, Soricelli A, Mascalchi M. 2011. Axial diffusivity is increased in the degenerating superior cerebellar peduncles of Friedreich's ataxia. Neuroradiology. 53(5):367-372.

Dürr A, Cossee M, Agid Y, Campuzano V, Mignard C, Penet C, Mandel JL, Brice A, Koenig M. 1996. Clinical and genetic abnormalities in patients with Friedreich's ataxia. N Engl J Med. 335(16):1169-1175.

Edden RA, Jones DK. 2011. Spatial and orientational heterogeneity in the statistical sensitivity of skeleton-based analyses of diffusion tensor MR imaging data. J Neurosci Methods. 201(1):213-219. 
Fortuna F, Barboni P, Liguori R, Valentino ML, Savini G, Gellera C, Mariotti C, Rizzo G, Tonon C, Manners D, Lodi R, Sadun AA, Carelli V. 2009. Visual system involvement in patients with Friedreich's ataxia. Brain. 132(Pt 1):116-123.

França MC Jr, D’Abreu A, Yasuda CL, Bonadia LC, Santos da Silva M, Nucci A, Lopes-Cendes I, Cendes F. 2009. A combined voxel-based morphometry and 1H-MRS study in patients with Friedreich's ataxia. J Neurol. 256(7):1114-1120.

Galaburda AM, LeMay M, Kemper TL, Geschwind N. 1978. Right-left asymmetries in the brain. Science. 199(4331):852-856.

Genovese CR, Lazar NA, Nichols T. 2002. Thresholding of statistical maps in functional neuroimaging using the false discovery rate. Neuroimage. 15(4):870878.

Georgiou-Karistianis N, Akhlaghi H, Corben, LA, Delatycki MB, Storey E, Bradshaw JL, Egan GF. 2012. Decreased functional brain activation in Friedreich ataxia using the Simon effect task. Brain Cogn. 79(3):2000-2008.

Gilman S, Junck L, Markel DS, Koeppe RA, Kluin KJ. 1990. Cerebral glucose hypermetabolism in Friedreich's ataxia detected with positron emission tomography. Ann Neurol. 28(6):750-757.

Ginestroni A, Diciotti S, Cecchi P, Pesaresi I, Tessa C, Giannelli M, Della Nave R, Salvatore E, Salvi F, Dotti MT, Piacentini S, Soricelli A, Cosottini M, De Stefano N, Mascalchi M. 2012. Neurodegeneration in friedreich's ataxia is associated with a mixed activation pattern of the brain. A fMRI study. Hum Brain Mapp. 33(8):1780-1791.

Gong G, He Y, Concha L, Lebel C, Gross DW, Evans AC, Beaulieu C. 2009a. Mapping anatomical connectivity patterns of human cerebral cortex using in vivo diffusion tensor imaging tractography. Cerebral Cortex. 19(3):524-536. 
Gong G, He Y, Evans AC. 2011. Brain connectivity gender makes a difference. The Neuroscientist. 17(5):575-591.

Gong G, Rosa-Neto P, Carbonell F, Chen ZJ, He Y, Evans AC. 2009b. Age- and gender-related differences in the cortical anatomical network. J Neuro. 29(50):15684-15693.

Hart RP, Kwentus JA, Leshner RT, Frazier R. 1985. Information processing speed in Friedreich's ataxia. Ann Neurol. 17(6):612-614.

He Y, Evans A. 2010. Graph theoretical modeling of brain connectivity. Current Opinion in Neurology. 23(4):341-350.

Jbabdi S, Johansen-Berg H. 2012. Tractography: Where Do We Go from Here? Brain Connecitivty. 1(3):170-183.

Junck L, Gilman S, Gebarski SS, Koeppe RA, Kluin KJ, Markel DS. 1994. Structural and functional brain imaging in Friedreich's ataxia. Arch Neurol. 51(4):349-355.

Jenkinson M, Smith SM. 2001. A global optimisation method for robust affine registration of brain images. Medical Image Analysis. 5(2):143-156.

Klockgether T. Handbook of ataxia disorders. CRC Press, New York, 2000.

Klopper F, Delatycki MB, Corben LA, Bradshaw JL, Rance G, Georgiou-Karistianis N. 2011. The test of everyday attention reveals significant sustained volitional attention and working memory deficits in Friedreich Ataxia. J Int Neuropsychol Soc. 17(1):196-200.

Koeppen AH. 2011. Friedreich's ataxia: pathology, pathogenesis, and molecular genetics. J Neurol Sci. 303(1-2):1-12.

Leemans A, Jones DK. 2009. The B-matrix must be rotated when correcting for subject motion in DTI data. Magn Reson Med. 61(6):1336-1349. 
Lynch DR, Farmer JM, Tsou AY, Perlman S, Subramony SH, Gomez CM, Ashizawa T, Wilmot GR, Wilson RB, Balcer LJ. 2006. Measuring Friedreich ataxia: complementary features of examination and performance measures. Neurology. 66(11):1711-1716.

Mascalchi M, Salvi F, Piacentini S, Bartolozzi C. 1994. Friedreich's ataxia: MR findings involving the cervical portion of the spinal cord. AJR Am J Roentgenol. 163(1):187-191.

Mascalchi M, Vella A. 2012. Magnetic resonance and nuclear medicine imaging in ataxias. Handb Clin Neurol. 103:85-110.

Mantovan MC, Martinuzzi A, Squarzanti F, Bolla A, Silvestri I, Liessi G, Macchi C, Ruzza G, Trevisan CP, Angelini C. 2006. Exploring mental status in Friedreich's ataxia: a combined neuropsychological, behavioral and neuroimaging study. Eur J Neurol. 13(8):827-835.

Nelson HE, Willison J, Owen AM. 1992. National adult reading test (2nd ed). Int J Geriatr Psychiatry. 7(7):533

Nichols TE, Holmes AP. 2001. Nonparametric permutation tests for functional neuroimaging: a primer with examples. Hum Brain Mapp. 15:1-25.

Ormerod IE, Harding AE, Miller DH, Johnson G, MacManus D, du Boulay EP, Kendall BE, Moseley IF, McDonald WI. 1994. Magnetic resonance imaging in degenerative ataxic disorders. J Neurol Neurosurg Psychiatry. 57(1):51-57.

Pagani E, Ginestroni A, Della Nave R, Agosta F, Salvi F, De Michele G, Piacentini S, Filippi M, Mascalchi M. 2010. Assessment of brain white matter fiber bundle atrophy in patients with Friedreich ataxia. Radiology. 255(3):882-889.

Puccio H, Simon D, Cossée M, Criqui-Filipe P, Tiziano F, Melki J, Hindelang C, Matyas R, Rustin P, Koenig M. 2001. Mouse models for Friedreich ataxia exhibit 
cardiomyopathy, sensory nerve defect and Fe-S enzyme deficiency followed by intramitochondrial iron deposits. Nat Genet. 27(2):181-186.

Rizzo G, Tonon C, Valentino ML, Manners D, Fortuna F, Gellera C, Pini A, Ghezzo A, Baruzzi A, Testa C, Malucelli E, Barbiroli B, Carelli V, Lodi R. 2011. Brain diffusion-weighted imaging in Friedreich's ataxia. Mov Disord. 26(4):705-712.

Rubinov M, Sporns O. 2010. Complex network measures of brain connectivity: uses and interpretation. Neuroimage 52(3):1059-1069.

Rushton WAH. 1951. Theory of the effects of fibre size in medulated nerve. J Physiol. 115:101-122.

Schmahmann JD, Sherman JC. 1998. The cerebellar cognitive affective syndrome. Brain. 121:561-579.

Schulz JB, Boesch S, Bürk K, Dürr A, Giunti P, Mariotti C, Pousset F, Schöls L, Vankan P, Pandolfo M. 2009. Diagnosis and treatment of Friedreich ataxia: a European perspective. Nat Rev Neurol. 5(4):222-234.

Simon D, Seznec H, Gansmuller A, Carelle N, Weber P, Metzger D, Rustin P, Koenig M, Puccio H. 2004. Friedreich ataxia mouse models with progressive cerebellar and sensory ataxia reveal autophagic neurodegeneration in dorsal root ganglia. J. Neurosci. 24:1987-1995.

Smith SM, Jenkinson M, Johansen-Berg H, Rueckert D, Nichols TE, Mackay CE, Watkins KE, Ciccarelli O, Cader MZ, Matthews PM, Behrens TE. 2006. Tractbased spatial statistics: voxelwise analysis of multi-subject diffusion data. Neuroimage. 31(4):1487-1505.

Sporns O, Tononi G Kotter R. 2005. The Human Connectome: A structural description of the human brain. PLoS Computational Biology. 1(4):e42.

Subramony SH, May W, Lynch D, Gomez C, Fischbeck K, Hallett M, Taylor P, Wilson R, Ashizawa T, Cooperative Ataxia Group. 2005. Measuring friedreich 
ataxia: Interrater reliability of a neurologic rating scale. Neurology. 64(7):12611262.

Tyszka JM, Kennedy DP, Adolphs R, Paul LK. 2011. Intact bilateral resting-state networks in the absence of the corpus callosum. J Neurosci. 31(42):1515415162.

Tzourio-Mazoyer N, Landeau B, Papathanassiou D, Crivello F, Etard O, Delcroix N, Mazoyer B, Joliot M. 2002. Automated anatomical labeling of activations in SPM using a macroscopic anatomical parcellation of the MNI MRI single-subject brain. Neuroimage. 15:273-289.

van den Heuvel MP, Hulshoff Pol HE. 2010. Exploring the brain network: a review on resting-state fMRI functional connectivity. Eur Neuropsychopharmacol. 20(8):519-534.

van den Heuvel MP, Mandl RC, Kahn RS, Hulshoff Pol HE. 2009. Functionally linked resting-state networks reflect the underlying structural connectivity architecture of the human brain. Hum Brain Mapp. 30(10):3127-3141.

van den Heuvel MP, Stam CJ, Boersma M, Hulshoff Pol HE. 2008. Small-world and scale-free organization of voxel-based resting-state functional connectivity in the human brain. Neuroimage. 3(3):528-539.

White M, Lalonde R, Botez-Marquard T. 2000. Neuropsychologic and neuropsychiatric characteristics of patients with Friedreich's ataxia. Acta Neurol Scand. 2000 102(4):222-226.

Wig GS, Schlaggar BL, Petersen SE. 2011. Concepts and principles in the analysis of brain networks. Ann N Y Acad Sci. 1224(1):126-146.

Wollmann T, Nieto-Barco A, Monton-Alvarez F, Barroso-Ribal J. 2004. Ataxia de Friedreich: analisis de parametros de resonancia magnetica y correlatos con el enlentecimiento cognitivo y motor. Rev. Neurol. 38 (3):217-222. 
Wüllner U, Klockgether T, Petersen D, Naegele T, Dichgans J. 1993. Magnetic resonance imaging in hereditary and idiopathic ataxia. Neurology. 43(2):318325.

Yakovlev PI, Rakic P. 1966. Patterns of decussation of bulbar pyramids and distribution of pyramidal tracts on two sides of the spinal cord. Transactions of the American Neurological Association. 91:3667-3367.

Zalesky A. 2011a. Moderating registration misalignment in voxelwise comparisons of DTI data: a performance evaluation of skeleton projection. Magn Reson Imaging. 29(1):111-125.

Zalesky A, Fornito A. 2009. A DTI-derived measure of cortico-cortical connectivity. 2009. IEEE Trans Med Imag. 28(7):1023-1036.

Zalesky A, Fornito A, Harding IH, Cocchi L, Yucel M, Pantelis C, Bullmore ET. 2010. Whole-brain anatomical networks: does the choice of nodes matter? Neurolmage. 50(3):970-983.

Zalesky A, Fornito A, Seal ML, Cocchi L, Westin C-F, Bullmore ET, Egan GF, Pantelis C. 2011. Disrupted axonal fiber connectivity in schizophrenia. Biol Psychiatry. 69(1):80-89. 


\section{Tables}

Table 1. Summary of diffusion MRI studies of brain anatomy in Friedreich ataxia.

Table 2. Sample characteristics for individuals with FRDA and controls (mean and standard deviation).

Table 3. Mean streamline counts and corresponding standard errors for individuals with FRDA and controls. The $p$-values are for a $t$-test assessing the null hypothesis of equality in the mean streamline count between groups. The 14 (out of 90) regions listed are those that survived correction for multiple comparisons.

\section{Captions}

Figure 1. A fractional anisotropy (FA) template was constructed in MNI152 space using an iterative registration scheme (see Data Preprocessing and Image Registration). The mean contribution from the FRDA group and the mean contribution from the control group was combined to construct the template. Between-group differences in FA were then assessed in a voxel-wise manner. FA was significantly lower $(p<0.001)$ in the FRDA group compared with controls in a cluster spanning portions of the brainstem, dentate nucleus and cerebellar peduncles. The color of voxels comprising the cluster is modulated by the $t$ statistic value. 
Figure 2. Fourteen of the 90 cortical and sub-cortical regions considered were connected with significantly fewer streamlines in the FRDA group, compared with controls ( $p<0.05$, false discovery rate corrected for 90 independent tests). Probabilistic streamlines were initiated from the between-group difference in fractional anisotropy (seed region). The total number of streamlines landing in each of 90 cortical and subcortical regions was enumerated and tested for a between-group difference.

Figure 3. The number of streamlines passing through each voxel was enumerated to construct super-resolution track density images (500 $\mu \mathrm{m})$. Track density images were then registered to MNI space and averaged over each group. Appreciable between-group differences are visually evident when comparing corresponding slices from the group-averaged track density images.

Figure 4. Estimates for the volume of cerebello-cerebral pathways were determined by binarizing the super-resolution track density images with a range of thresholds. The group-averaged volume estimates for the FRDA group (red) and the control group (blue) was plotted as a function of the threshold $(\mathbf{A})$. The estimated volume was significantly lower $(p<0.001)$ in the FRDA for all thresholds evaluated. The group-averaged anatomical location of the binary clusters from which volume was estimated is shown for two representative thresholds: 4000 (B) and 6000 (C).

Figure 5. Scatter plots representing the negative correlation between age of disease onset in the FRDA group and volume of cerebello-cerebral pathways. Volume was estimated by binarizing the super-resolution track density images at a range of thresholds: 8000 (A), 10,000 (B) and 12,000 (C). Solid lines represent lines of best fit. 


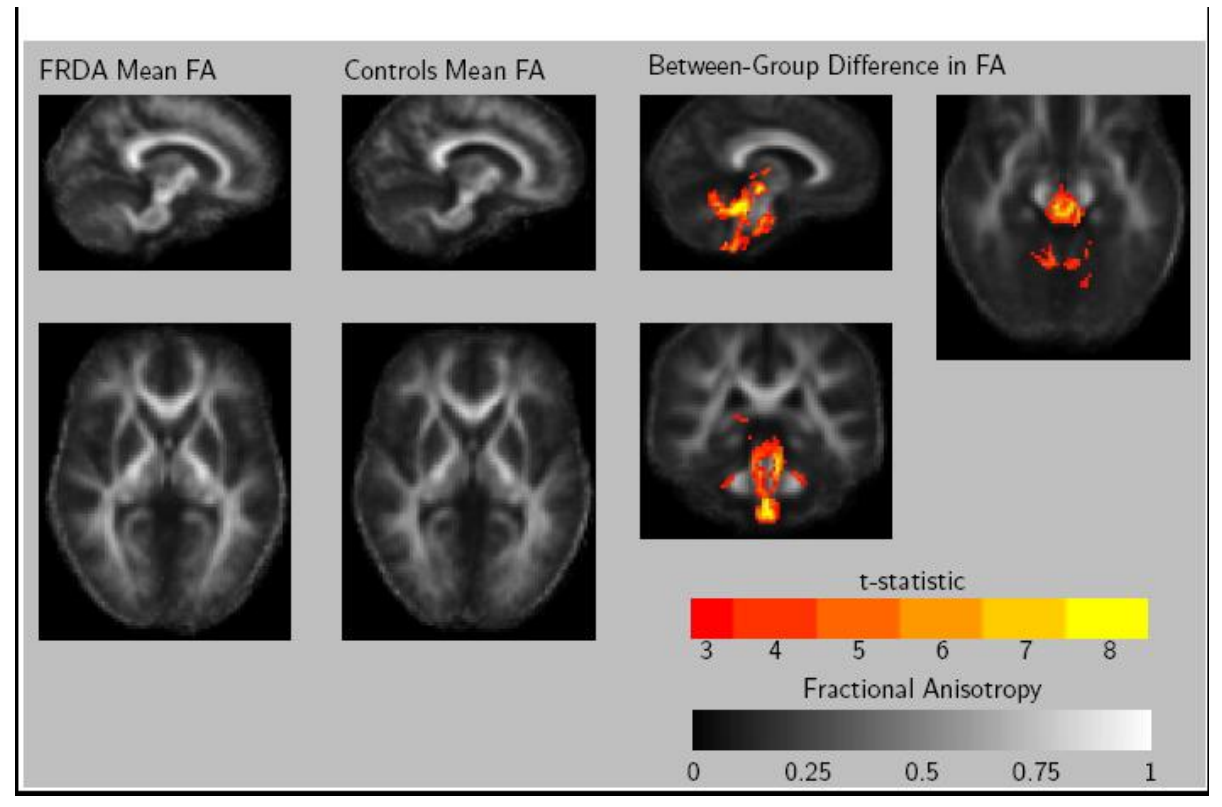




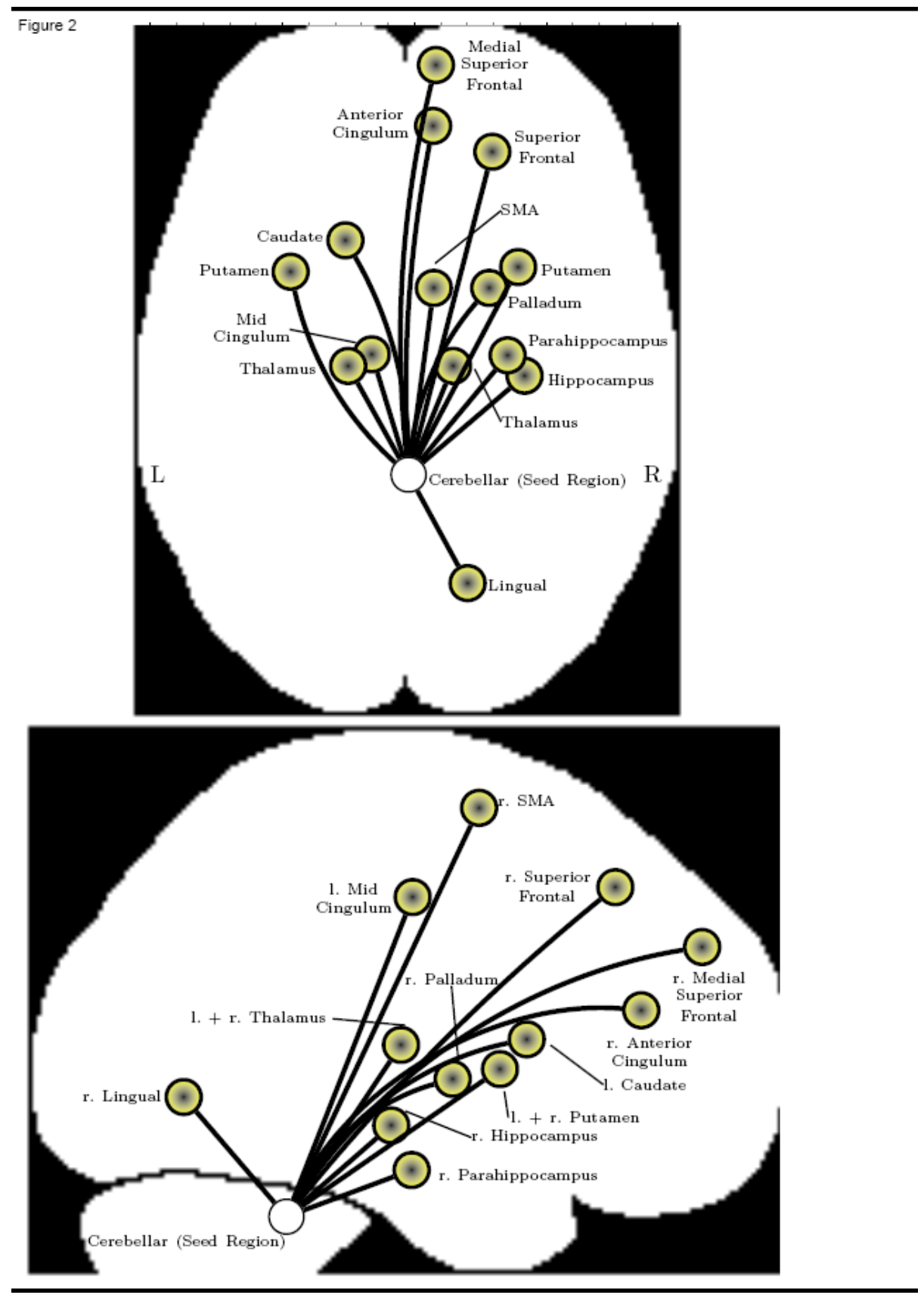



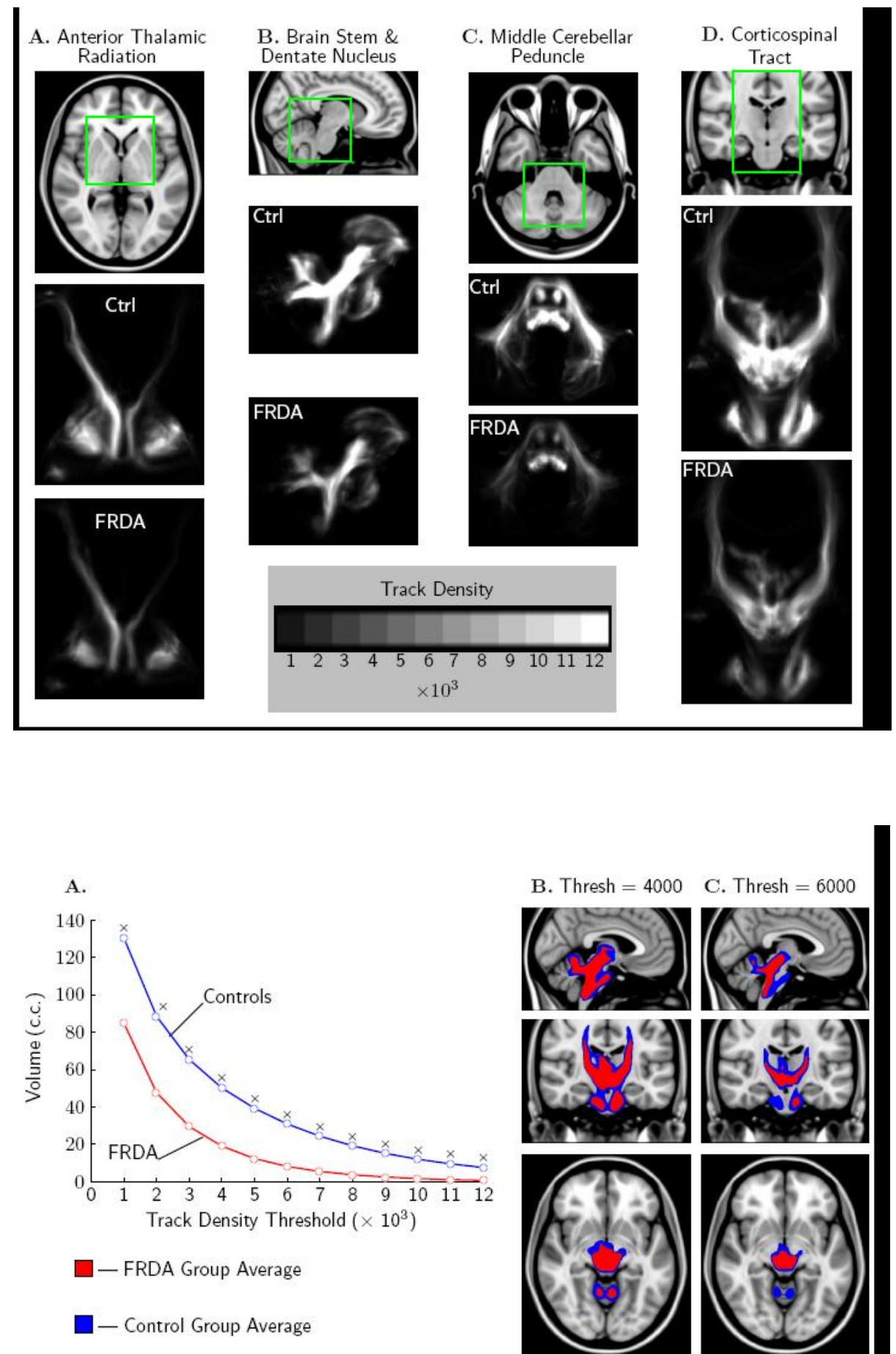

B. Thresh $=4000 \quad$ C. Thresh $=6000$

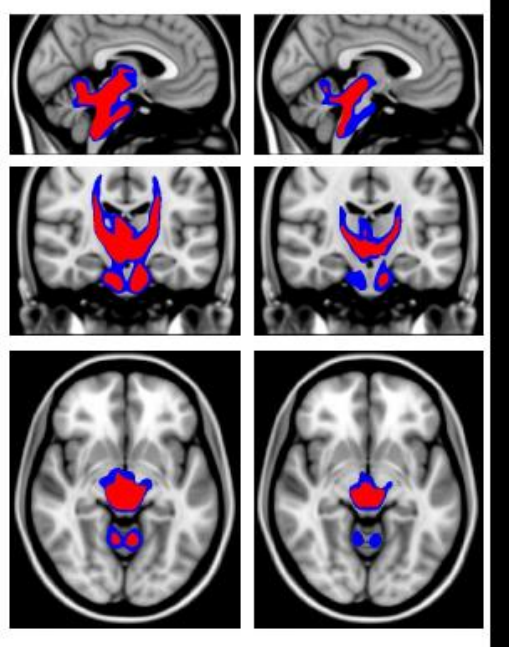




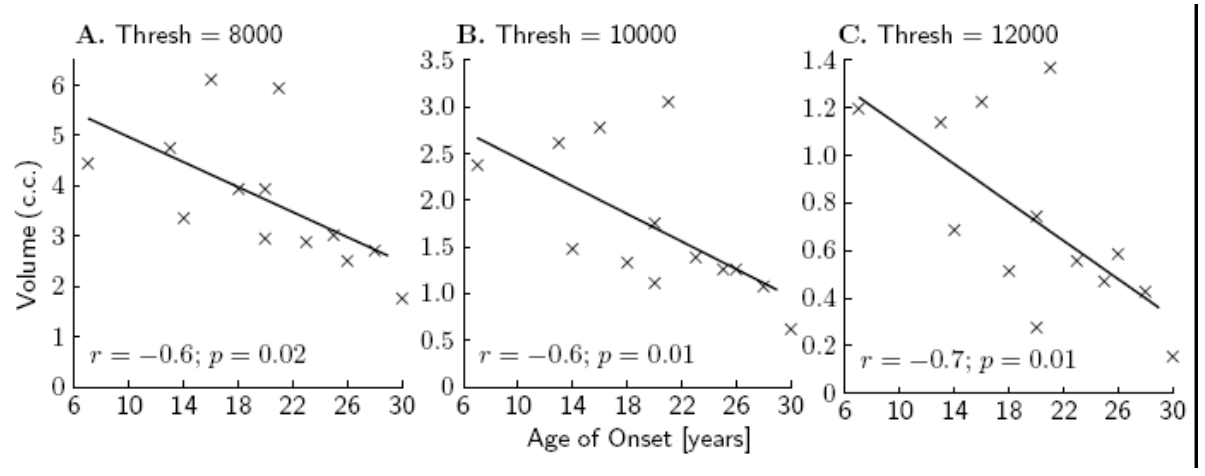




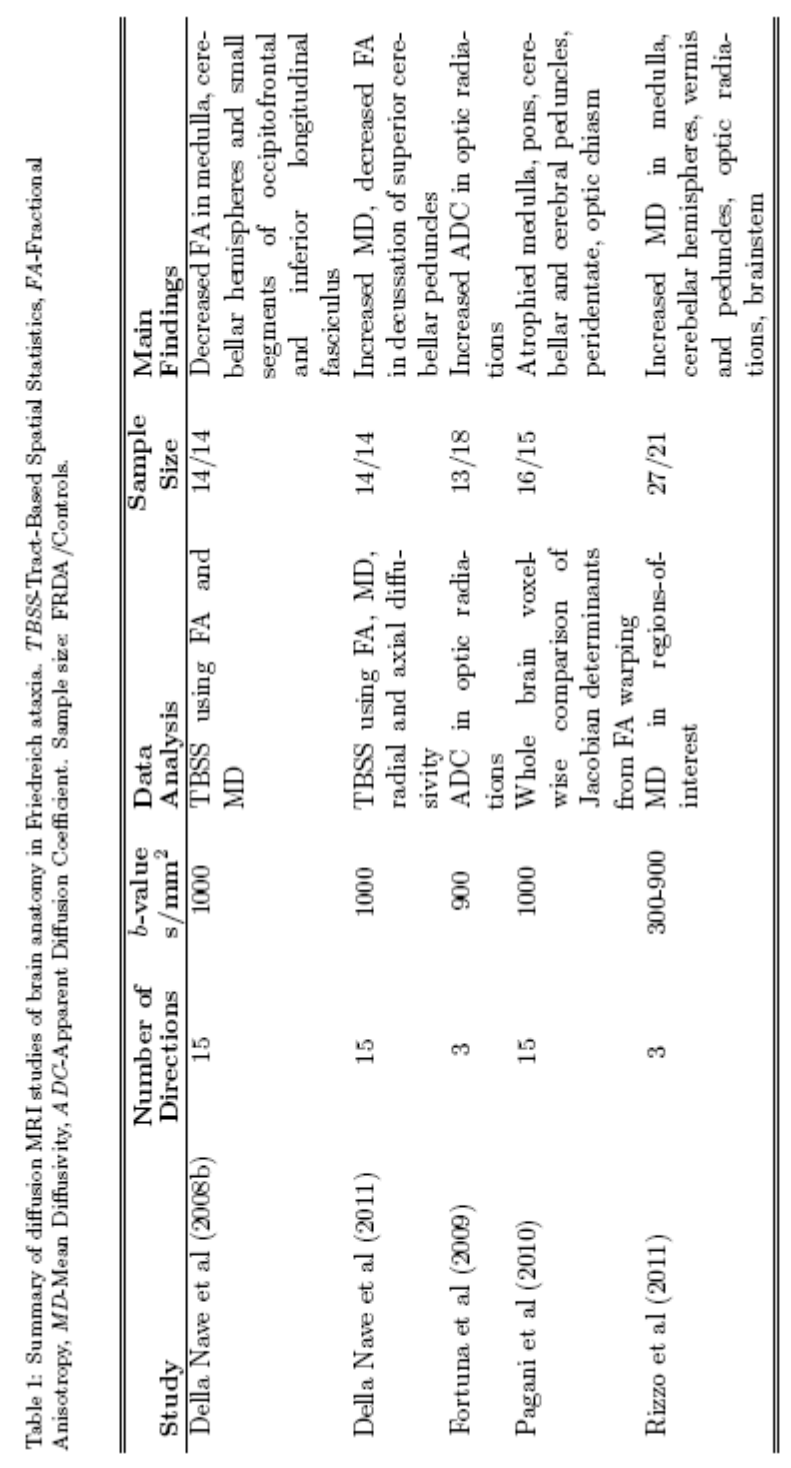


Table 2: Sample characteristics for individuals with FRDA and controls (mean and standard deviation).

\begin{tabular}{|c|c|c|}
\hline Measure & $\begin{array}{c}\text { FRDA } \\
n=13\end{array}$ & $\begin{array}{c}\text { Controls } \\
n=14\end{array}$ \\
\hline Age [years] & $35.5(9.4)$ & $33.7(7.9)$ \\
\hline NART ${ }^{a}$ & $117.1(3.1)$ & $121.5(2.6)$ \\
\hline Gender (males) & 6 & 9 \\
\hline BDI score $e^{b}$ & $7.0(7.9)$ & $3.7(4.7)$ \\
\hline FARS $^{c}$ & $86.5(15.7)$ & - \\
\hline Duration of Illness [years] & $15.9(5.9)$ & - \\
\hline Age of Onset [years] & $20(6.5)$ & - \\
\hline GAA repeat $^{d}$ & $608(243)$ & - \\
\hline
\end{tabular}

${ }^{a}$ NART: National Adult Reading Test

${ }^{b}$ BDI: Beck Depression Inventory.

${ }^{c}$ FARS: Friedreich Ataxia Rating Scale.

${ }^{d}$ GAA: FXN GAA repeat size of smaller allele.

Table 3: Mean streamline counts and corresponding standard errors for individuals with FRDA and controls. The $p$-values are for a t-test assessing the null hypothesis of equality in the mean streamline count between groups. The 14 (out of 90 ) regions listed are those that survived correction for multiple comparisons.

\begin{tabular}{l|ccc}
\hline \hline & FRDA & Controls & \\
Region & Streamline Count & Streamline Count & $p$-value \\
\hline L. Thalamus & $374 \pm 9$ & $535 \pm 22$ & $<0.0001$ \\
R. Thalamus & $706 \pm 12$ & $875 \pm 19$ & $<0.0001$ \\
R. Parahippocampal & $37 \pm 2$ & $64 \pm 6$ & 0.0001 \\
R. Putamen & $75 \pm 10$ & $146 \pm 14$ & 0.0003 \\
R. Superior Medial Frontal & $7 \pm 1$ & $20 \pm 3$ & 0.001 \\
R. Lingual & $90 \pm 8$ & $175 \pm 19$ & 0.004 \\
L. Caudate & $139 \pm 14$ & $235 \pm 15$ & 0.002 \\
R. Superior Frontal & $39 \pm 5$ & $82 \pm 9$ & 0.002 \\
L. Putamen & $20 \pm 4$ & $39 \pm 7$ & 0.003 \\
R. Pallidum & $72 \pm 12$ & $125 \pm 11$ & 0.004 \\
R. SMA & $34 \pm 4$ & $69 \pm 9$ & 0.005 \\
R. Anterior Cingulum & $2 \pm 1$ & $10 \pm 2$ & 0.007 \\
R. Hippocampus & $51 \pm 4$ & $78 \pm 5$ & 0.01 \\
L. Mid Cingulum & $38 \pm 8$ & $66 \pm 9$ & 0.01 \\
\hline \hline
\end{tabular}

